\title{
EFFICIENT RECONSTRUCTIONS OF COMMON ERA CLIMATE VIA INTEGRATED NESTED LAPLACE APPROXIMATIONS.
}

\author{
LUIS A. BARBOZA, JULIEN EMILE-GEAY, BO LI, AND WAN HE
}

\begin{abstract}
Paleoclimate reconstruction on the Common Era (1-2000AD) provide critical context for recent warming trends. This work leverages integrated nested Laplace approximations (INLA) to conduct inference under a Bayesian hierarchical model using data from three sources: a state-of-the-art prox database (PAGES 2k), surface temperature observations (HadCRUT4), and latest estimates of external forcings. INLA's computational efficiency allows to explore several model formulations (with or without forcings, explicitly modeling internal variability or not), as well as five data reduction techniques. Two different validation exercises find a small impact of data reduction choices, but a large impact for model choice, with best results for the two models that incorporate external forcings. These models confirm that man-made greenhouse gas emissions are the largest contributor to temperature variability over the Common Era, followed by volcanic forcing. Solar effects are indistinguishable from zero. INLA provide an efficient way to estimate the posterior mean, comparable with the much costlier Monte Carlo Markov Chain procedure, but with wider uncertainty bounds. We recommend using it for exploration of model designs, but full MCMC solutions should be used for proper uncertainty quantification.
\end{abstract}

\section{INTRODUCTION.}

Earth's climate presents a continuum of variability, with periodic and non-periodic fluctuations ranging from 1 to $10^{8}$ years (Pelletier, 1998). In particular, variability on scales of decades to centuries is of paramount importance for adaption and planning to anthropogenic climate change, yet is incompletely sampled by the relatively short historical record of wide-spread instrumental observations, going back to about $1850 \mathrm{CE}$ (Masson-Delmotte and et al., 2013). It is thus critical to reconstruct these variations from the paleoclimate

Date: August 10, 2021.

Key words and phrases. Hierarchical Bayesian Model, INLA, Paleoclimate Reconstruction. 
record as quantitatively as possible. A particular focus has been reconstructions of global or hemispheric temperature from high-resolution proxy observations (Jones and et al., 2009).

Many methods have been developed to reconstruct past climates, particularly temperatures: principal component regression (Mann et al., 1998, Luterbacher et al., 2004), regularized forms of the expectation-maximization algorithm (Schneider, 2001; Mann et al., 2007, Emile-Geay et al., 2013, Guillot et al., 2015), canonical correlation analysis (Smerdon et al. 2010; Wang et al., 2014), pairwise comparison (Hanhijärvi et al., 2013), data assimilation (Lee et al., 2008; Hakim et al., 2016), and Bayesian hierarchical models (BHMs).

BHMs offer several distinct advantages for paleoclimatic reconstruction. They can (i) treat different sources of uncertainty in a natural way, (ii) incorporate prior knowledge of the system in a logically-coherent manner, and (iii) allow for both inference and prediction (Tingley et al., 2012). Many studies have employed BHMs (e.g. Li et al., 2010, Tingley and Huybers, 2013; Barboza et al., 2014, hereafter, "B14")); some have used space-state schemes to linearly relate information that comes from paleoclimate observations together with information about external climatic forcings, resulting from well-mixed greenhouse gases, volcanic activity, and variations in solar output. Three problems arise in this case: the need to reduce dimensionality, the complexity/realism of the model, and the execution time of the numerical procedure.

B14 proposed a method that jointly models the variability of the temperature series (as a latent process) as well as the variability of those climatic and biological observations that serve as approximations of this process ("proxies"). The authors found that long memory error terms are necessary in absence of external forcing information within a BHM, but the presence of external forcing information substantially improves the reconstruction. The scope of the study was restricted by the computational requirements of the Monte Carlo Markov Chain (MCMC) procedure, which limited the sensitivity analysis and forced severe levels of data reduction.

In this article we extend the work of B14 by leveraging Integrated Nested Laplace Approximations (INLA). Doing so lightens the computational burden, which allows to (a) explore 
new model designs inspired by the physics of the problem; (b) consider various choices for data reduction; and (c) take the non-stationary nature of the observational network into account. In addition, this work makes use of the latest estimates of radiative forcing, as well as a state-of-the-art, open-access compilation of community-curated paleoclimate observations (PAGES2k Consortium, 2017). This ensures that our calculations are using the best available data and are completely reproducible.

The article is organized as follows: we start by describing the datasets (section 2), and the methodology (section 3). Results are then presented in section 4, discussed in Section 5, before concluding in section 6 .

\section{Datasets.}

2.1. Proxy data. Reconstructions of past climates rely on "proxies": indirect observations of climate, as recorded in borehole, coral, documentary, glacier ice, lake and marine sediment, sclerosponge, speleothem and tree-ring archives (Jones and et al., 2009). The PAGES2k global multiproxy database is a community-driven effort to synthesize all publicly-archived, temperature-sensitive proxy records of the past 2,000 years (PAGES2k Consortium, 2017). The most recent effort gathers 692 records from 648 locations, including all continental regions and major ocean basins. The records are from trees, ice, marine and lake sediments, corals, speleothems, and documentary evidence. They range in length from 50 to 2000 years, with a median of 547 years, while temporal resolution ranges from biweekly to centennial. The vast majority of records are annually-resolved, with minimal dating uncertainty. Here, the data used have been mapped to a standard normal using the method of van Albada and Robinson (2007). The data are available in a standard format (LiPD) readable in R, Python and Matlab, to ensure reproducible workflows (McKay and Emile-Geay, 2016).

Each of those proxies has different time horizons (Fig. 1, bottom), which creates challenges for inference. Unlike previous studies (e.g. B14), we strive to take into account the information available in most proxies, despite their temporal diversity. In order to select proxies with high predictive power, we first chose those series with large correlations with respect to 

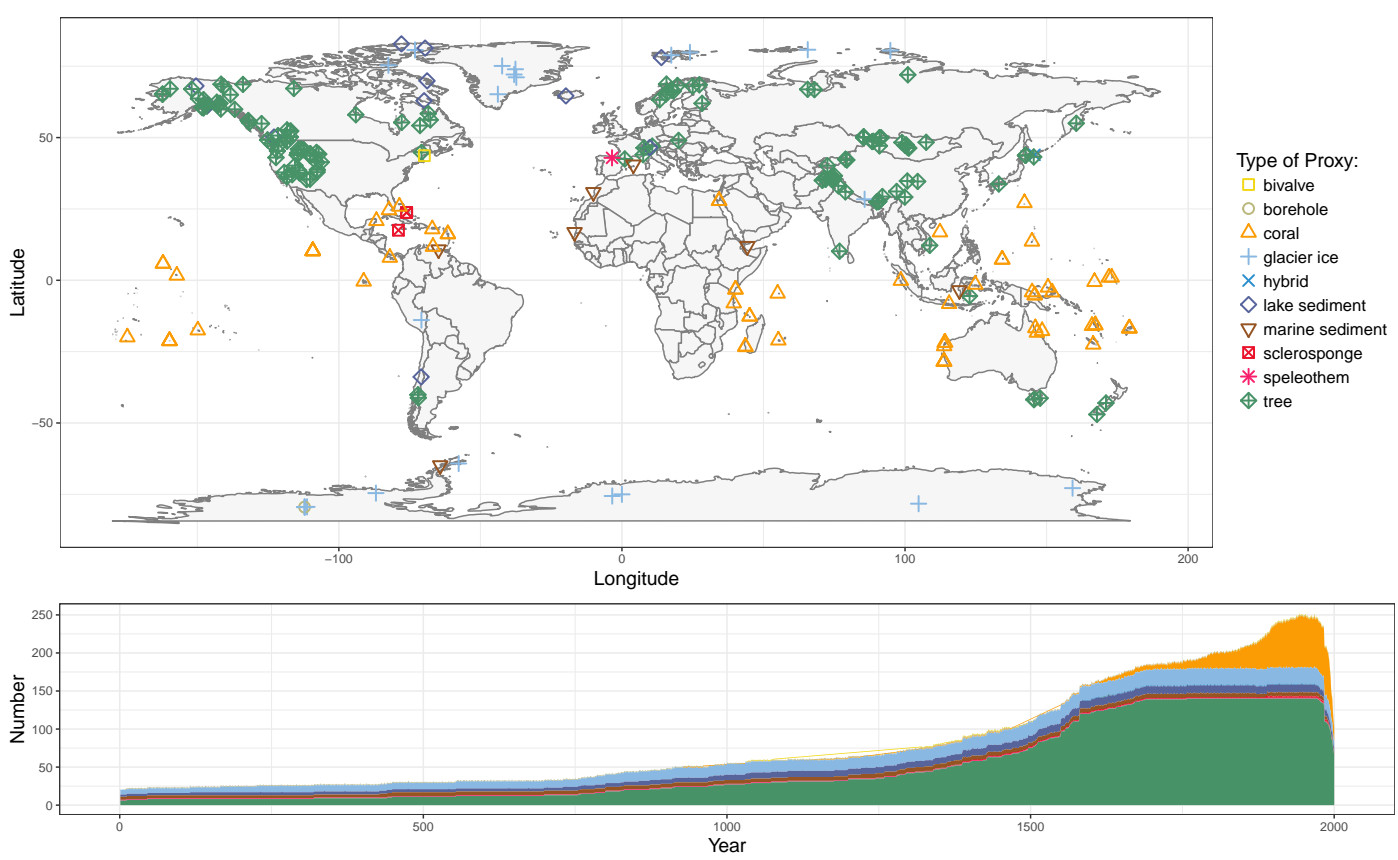

FIGURE 1. Distribution and temporal availability of PAGES2k proxies, after applying the screening procedure PAGES2k Consortium (2017).

their closest spatial temperature record in the HadCRUT4.2 dataset (Morice et al., 2012). More details on this "screening" procedure, which controls for the multiple test problem (Benjamini and Hochberg, 1995), can be found in PAGES2k Consortium (2017); it whittles down the database to 257 proxies (Fig. 1).

\begin{tabular}{c|c|c}
\hline Group & Interval (year AD) & Number of Proxies \\
\hline 1 & $1-250$ & 19 \\
2 & $251-500$ & 25 \\
3 & $501-750$ & 29 \\
4 & $751-1000$ & 33 \\
5 & $1001-1250$ & 54 \\
6 & $1251-1500$ & 65 \\
7 & $1501-1750$ & 105 \\
8 & $1751-2000$ & 146 \\
\hline
\end{tabular}

TABLE 1. Distribution of proxies according to their temporal availability.

Due to the diversity of start dates in the proxies database (Fig. 1), we gather proxies into non-homogeneous groups where each group has temporal availability within a $250 \mathrm{y}$ interval (Table 1). Proxy series whose proportion of missing annual observations is larger than 5\% during the calibration period (1900-2000, as in B14) are excluded. 
2.2. Temperature data. We estimate Global Mean Surface Temperature (GMST) from the HadCRUT4 global temperature dataset provided by the Met Office Hadley Centre and the Climatic Research Unit at the University of East Anglia, UK (version 4.4.0.0). The dataset consists of instrumental, in situ observations of surface temperature over land (Jones et al. 2012 ) and ocean (Kennedy et al. 2011a b). The observations are expressed as anomalies relative to the monthly-mean seasonal cycle over the 1961-1990 period, in degrees Celsius. Though HadCRUT4 features a rather sophisticated analysis of error sources (Morice et al., 2012), we neglect these uncertainties against the much larger uncertainties affecting paleoclimate observations, and simply use the median estimate, averaged on an annual basis.

2.3. Forcing data. These use the most recent compilations from the PMIP4-CMIP6 project (Jungclaus and et al., 2017):

Volcanic forcing from the evolv2k dataset (Toohey et al., 2016), which reconstructed zonal mean aerosol optical depth at $550 \mathrm{~nm}$, covering the $500 \mathrm{BCE}$ to $1900 \mathrm{CE}$ time period. For 1900 (or 1850) to present, Thomason et al. (2015) is used to fill in the forcing table. The data were given as a function of latitude, so were area-weighted and averaged to form a global estimate (Fig. 2, top).

Solar forcing data is computed from the SATIRE-H dataset (Vieira et al., 2011). Irradiance is provided on a decadal basis from $9495 \mathrm{BC}$ - 1939AD and then on a daily basis from 1940AD onwards. We interpolated the data at annual resolution using BSplines (Fig. 2, middle).

Greenhouse-Gases concentrations: hemispheric means of mole fraction of carbon dioxide in air (ppm) with annual resolution (Meinshausen and et al., 2017) as well as ice core measurements prior to that date (Jungclaus and et al., 2017) (Fig. 2, bottom).

\section{Methodology}

3.1. Data reduction methods. For some intervals in Table 1 , the proxy data matrix is large compared to the 101 yearly samples used to train the model (1900-2000). This "large $p$, small $n$ " problem calls for some form of data reduction. Following B14, we generate a set of "Reduced Proxy" ( $R P)$ time series, which condense individual proxy time series into a single 

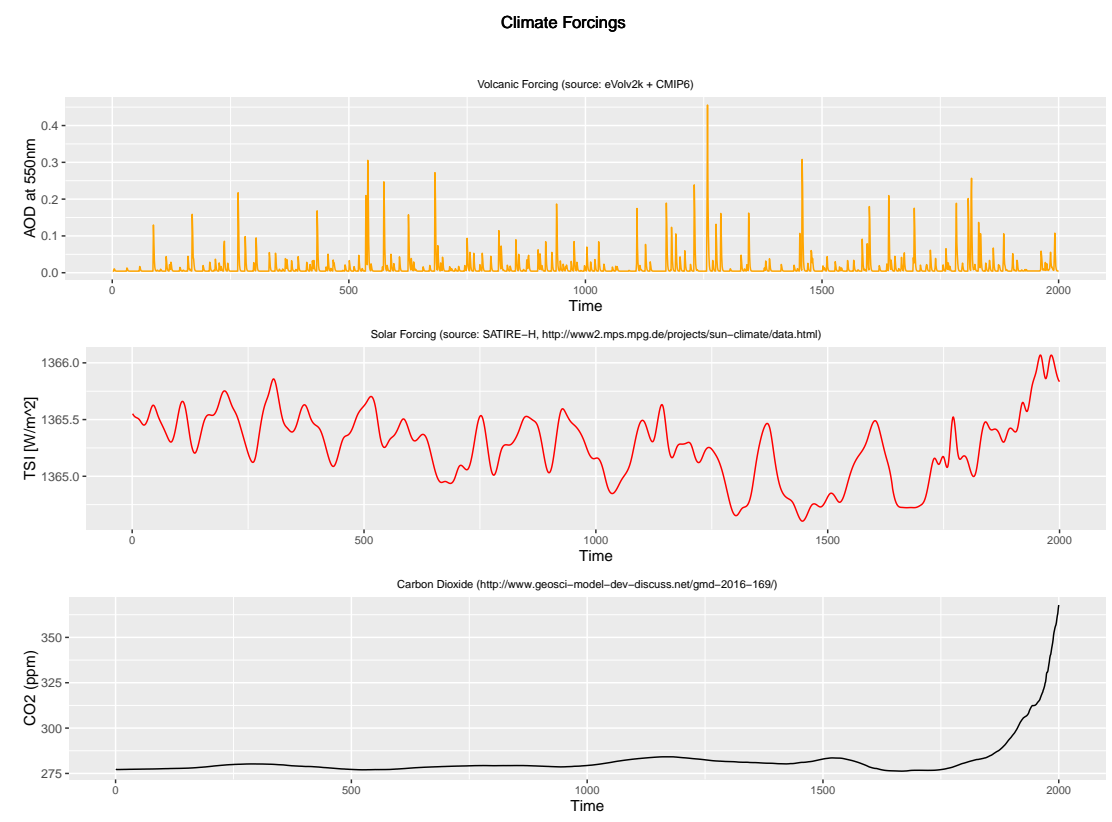

Figure 2. Main climate Forcings of the Common Era (1-2000 AD): volcanic, solar, and carbon dioxide. AOD $=$ Aerosol optical depth. TSI $=$ total solar irradiance. $\mathrm{ppm}=$ parts per million. See text for details.

time series with larger predictive power over the GMST target. Since this is a critical choice to make, we extend B14 by carefully investigating five common data reduction methods:

Lasso Regression (LR) The Lasso regression penalizes the usual sum of squares with an argument containing the sum of the absolute values of each coefficient in the classical linear regression model, multiplied by an additional smoothing parameter (Tibshirani, 1996). Due to the geometric nature of the term of penalization, the search of estimators tends to assign values very close to zero to variables that have almost null effects with respect to the dependent variable, which makes the resulting models easily interpretable. This method is very common to data reduction and easy to implement, but it often tends to select overly complex models, that is, it tends to show "false positives" in the variable selection process (Fan and Lv, 2010). The Lasso may also run into inconsistency issues when the variables are highly correlated (Zou and Hastie, 2005). We used 10-fold cross validation to select the smoothing parameter when we carried out Lasso regression (see Tibshirani (1996) for more details). 
Sparse Partial Least Squares (sPLS) Partial least squares seek to reduce the highdimensionality issues of the design matrix in linear regression models through a latent matrix whose columns maximize the product of the linear correlation between predictors and responses and the variance of responses. The sPLS method further introduces sparseness to the partial least squares estimators by means of a $L_{1}$-penalty with a thresholding parameter, in order to avoid inconsistencies when there is a substantial number of noisy covariates Chun and Keleş, 2010; Chung et al., 2013). However, this method is inefficient in measuring the statistical significance of whether the parameters associated with certain variables are effectively zero (Olson Hunt et al., 2014). In our implementation, the thresholding parameter involved in sPLS is estimated using a 10-fold cross-validation criteria.

Sliced Inverse Regression (SIR) with CSS selection In general, SIR methods (Li, 1991) reduce the excess dimension in a non-parametric setting through the estimation of the linear space spanned by the coefficients of the covariables, also known as effective dimension reduction (EDR) subspace. This subspace is obtained by an approximate eigenvalue decomposition that involves an estimation of the covariance matrices of the design matrix and the conditional expectation of the explanatory variables given the response. The estimation of the covariance matrix requires to partition the dependent variable into subgroups, called slices. The SIR method can capture both linear or nonlinear associations between the response and covariates. However, the estimation of the dimension reduction space does not actually lead to a variable selection procedure and the covariance estimation relies heavily on the homogeneity of the response within each slice (Wu et al., 2010). Because of this, we opt to incorporate the CSS (Closest submodel selection) variable selection procedure into the SIR method. Furthermore, to better deal with the fact that there is a larger number of covariates than observations, we employed the SIR-QZ algorithm, an upgrade of the SIR method based on the generalized Shur decomposition for underdetermined cases Coudret et al., 2014, 2017). Finally, we studied the association between proxies and temperatures 
through a linear regression between the observed anomalies and a number of EDR directions, i.e. an orthogonal basis of the EDR subspace, determined by marginal dimension tests Cook, 2004).

Principal Component Regression (PCR) PCR simply means that we replace the original covariates by their PCs. To select how many and which PCs to use, we fitted a regression model between the temperature and PCs of eight different sets of covariates selected in each of the eight nests (Table 1) based on the training data from the calibration period. We then selected the number of PCs in each of the eight regressions as the minimum number that attains, for the first time, an adjusted $R^{2}$ of at least $70 \%$ in each case. PCR is often used when covariates are highly-correlated or when the number of covariates is larger than the number of observations. A caveat of this method is that the principal components with smaller contribution to variance are not necessarily the ones that associate less with the dependent variable in a linear regression model (Jolliffe, 1982; Tibshirani, 1996).

Supervised Principal Components (sPCR) Because of the above-mentioned caveat of directly using PCR, Bair et al. (2006) developed a technique where PCR is applied only to a certain subset of covariates that exhibits a considerable amount of association with the dependent variable, and the threshold of "considerable" is chosen through cross-validation. Compared to PCR, the sPCR ensures the dimensionality reduction on the covariates space while taking the association between the covariates and the dependent variable into account. In general, its performance is quite similar to sPLS Chung et al., 2013).

The data reduction allows us to fit linear regression models between temperatures and proxies. Reducing proxies not only eases the computation, but also makes the reconstruction less uncertain by removing the part of noise in proxies that is related to local temperatures. After we fit a linear regression model under each of the five data reduction methods and for each of the eight proxy groups listed in Table 1, we compute a single reduced proxy series following B14 for each group. All reduced proxies are shown in Fig. 3. These series are highly correlated, with the reduced proxies obtained by PCR standing out as least similar to the others by this metric (Fig. 4). 


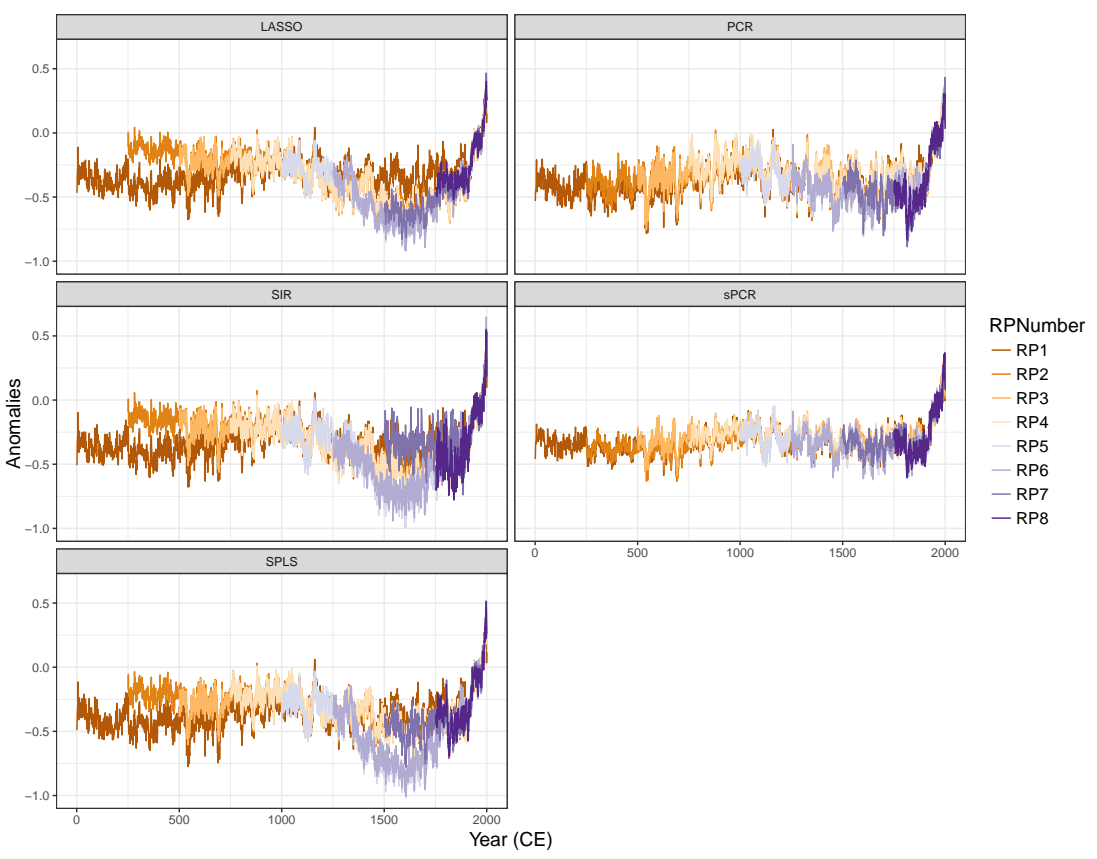

Figure 3. Reduced Proxies among methods.

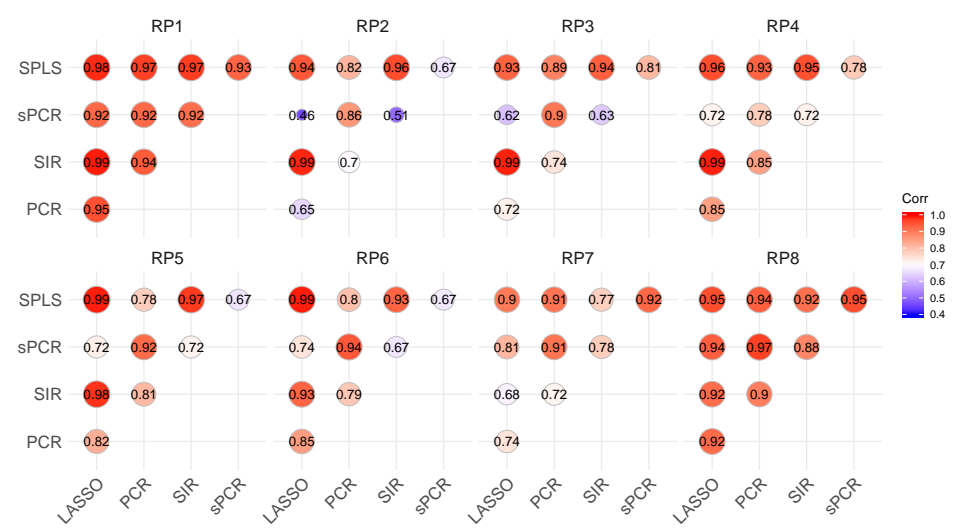

Figure 4. Correlation Matrices among 5 different Reduced Proxy series

3.2. Model Specification. The first level of a BHM always models the likelihood of the data (Tingley et al., 2012). The second level models the temperature process, and the third models observational uncertainties.

As in Li et al. (2010) and B14, our process model includes radiative forcings, since they are known to drive the temperature process. This also allows to attribute forcings (i.e., determine causality, Hegerl and Zwiers, 2011) as part of the inference procedure. However, this raises the spectre of overfitting, whereby the model would discount the noisy proxy data 
and place undue weight on the forcings, and not enough on internal variability. To mitigate this risk, it may be preferable to model temperature fluctuations as a smooth function of time (say, via splines) without including forcings, then perform forcing attribution on the inferred temperature posterior (Schurer et al. 2013a b), which guarantees independence: this way, if the reconstruction bears a strong resemblance to the forcings, it is only because the latter are reflected in the values of the predictors, not because they were fed to the model. In this section we explore both end-members, as well as an intermediate case. We first define:

- $R P_{t}^{i}: i$-th reduced proxy at time $t$.

- $T_{t}$ : temperature anomaly at time $t$.

- $\tilde{C}_{t}=\log \left(C_{t}\right)$ : Transformed greenhouse gases. The log transformation is chosen to approximate the radiative forcing due to changes in the equivalent $\mathrm{CO}_{2}$ concentration (see B14).

- $\tilde{V}_{t}=\log \left(-V_{t}+1\right)$ : Transformed volcanic forcing. See more details in B14 for the choice of the transformation.

- $B_{t}^{k, \tau}: k$-th B-Spline basis function at time $t$ with a uniform knot sequence $\tau$ de Boor. 2001: Ramsay and Silverman, 2005). Here we choose cubic B-spline bases and we denote $K(\tau)$ as the total number of basis elements.

We then define the first level of BHM as $R P_{t}^{i}=\alpha_{0}^{i}+\alpha_{1}^{i} T_{t}+\epsilon_{t}^{i}$, where $\left\{\alpha_{j}^{i}\right\}$ are intercepts $(j=0)$ and slopes $(j=1)$ for $i=1, \ldots, N$, and $\epsilon_{t}^{i}$ are normally-distributed random variables with finite variances $\left\{\sigma_{i}^{2}\right\}$. Note that in our case $N=8$; the time variable $t$ is defined on each nest according to the intervals of Table 1. We explore three formulations of the process level:

No forcing (model "NF") The main idea of this model is to provide a baseline that ignores external forcings. To capture low-frequency behavior, we include a smoothing function as:

$$
T_{t}=\beta_{0}+\sum_{k=1}^{K(\tau)} \beta_{k} B_{t}^{k, \tau}+\eta_{t}
$$


where $\beta_{k}$ are coefficients for B-spline bases, and $\eta_{t}$ are also normally-distributed random variables with finite variances $\sigma_{\eta}^{2}$. For simplicity, the error terms $\epsilon_{t}^{i}$ and $\eta_{t}$ are assumed to be independent.

With forcing (model "WF") Like B14, this model explicitly models temperature as linearly driven by radiative forcing:

$$
T_{t}=\beta_{0}+\beta_{1} S_{t}+\beta_{2} \tilde{V}_{t}+\beta_{3} \tilde{C}_{t}+\eta_{t}
$$

Note that Models NF and WF simply assume an IID error structure. This is because B14 found that complex error structures make little difference when forcings are added to the reconstruction and if we allow for a more flexible $\operatorname{AR}(1)$ structure for the error terms, the conclusions remain the same.

"Mixed" Model Finally we consider the more realistic case where temperature reflects both external forcings and internal dynamics. This model is a combination of equations (1) and (2), as follows:

$$
T_{t}=\beta_{0}+\beta_{1} S_{t}+\beta_{2} \tilde{V}_{t}+\beta_{3} \tilde{C}_{t}+\sum_{k=1}^{K(\tau)} \gamma_{k} B_{t}^{k, \tau}+\eta_{t}
$$

where $\gamma_{k}$ are the coefficients for the B-Spline basis. This last case is most relevant to climate dynamicists, as it explicitly represents both endogenous and exogenous variability. The downside is that the model is more complex, thus making estimation more challenging.

3.3. Computing posteriors with INLA. The computational challenge of MCMC inference has been a concern for Bayesian paleoclimate reconstructions. It is crucial to overcome this bottleneck before we can move forward to a more complex space-time reconstruction. Here we introduce the INLA sampling strategy to accelerate the MCMC procedure, as a proof of concept for more comprehensive models. The INLA approach is applicable to a general specification for which the mean $\eta_{i}$ of the observations $y_{i}$ follows a linear structure: 


$$
\eta_{i}=\alpha+\sum_{m-=1}^{M} \beta_{m} x_{m i}+\sum_{l=1}^{L} f_{l}\left(z_{l i}\right)
$$

where $\alpha$ represents an intercept, the coefficients $\beta=\left(\beta_{1}, \ldots, \beta_{M}\right)$ relate $M$ covariates $\left(x_{1}, \ldots, x_{M}\right)$ to $\eta_{i}$, and $f=\left\{f_{1}(\cdot), \ldots, f_{L}(\cdot)\right\}$ is a collection of random effects defined on a set of $L$ covariates $\left(z_{1}, \ldots, z_{L}\right)$ (see Rue et al. (2009) and Blangiardo et al. (2013)). Denote the set of random variables as $\theta=(\alpha, \beta, f)$ with $K$ hyperparameters $\psi=\left\{\psi_{1}, \ldots, \psi_{K}\right\}$, and the vector of observations as $y=\left(y_{1}, \ldots, y_{n}\right)$. Model (4) leads to conditional independence of $y$ given $\theta$ and $\psi$ :

$$
p(y \mid \theta, \psi)=\prod_{i=1}^{n} p\left(y_{i} \mid \theta_{i}, \psi\right)
$$

In our models, if we consider $y_{i}$ as the reduced proxies, $\eta_{i}$ the linear mean of the reduced proxies, $x_{m}$ the external forcings and/or the set of spline basis, and $f\left(z_{l}\right)$ the latent variables (temperature anomalies in our case), then our models fall into the general specification of INLA. The main objectives of our Bayesian estimation are to compute the marginal posterior distribution of each parameter in $\theta$ :

$$
p\left(\theta_{i} \mid y\right)=\int p\left(\theta_{i}, \psi \mid y\right) d \psi=\int p\left(\theta_{i} \mid \psi, y\right) p(\psi \mid y) d \psi
$$

To attain computational advantages, INLA assumes that the prior of vector $\theta$ is a multivariate normal random vector with a precision matrix that depends on hyperparameters $\psi$. INLA further approximates the two components $p(\psi \mid y)$ and $p\left(\theta_{i} \mid \psi, y\right)$. The first component is replaced by its a Laplace Approximation (see Tierney and Kadane (1986)):

$$
p(\psi \mid y)=\left.\frac{p(\theta, \psi \mid y)}{p(\theta \mid \psi, y)} \propto \frac{p(\psi) p(\theta \mid \psi) p(y \mid \theta)}{p(\theta \mid \psi, y)} \approx \frac{p(\psi) p(\theta \mid \psi) p(y \mid \theta)}{\tilde{p}(\theta \mid \psi, y)}\right|_{\theta=\theta^{*}(\psi)}:=\tilde{p}(\psi \mid y)
$$


where $\tilde{p}(\theta \mid \psi, y)$ is the Gaussian approximation of $p(\theta \mid \psi, y)$ and $\theta^{*}(\psi)$ is its mode (see Rue et al. (2009) ). The second component can be approximated in a similar way:

$$
p\left(\theta_{i} \mid \psi, y\right)=\left.\frac{p\left(\left(\theta_{i}, \theta_{-i}\right) \mid \psi, y\right)}{p\left(\theta_{-i} \mid \theta_{i}, \psi, y\right)} \approx \frac{p\left(\left(\theta_{i}, \theta_{-i}\right) \mid \psi, y\right)}{\tilde{p}\left(\theta_{-i} \mid \theta_{i}, \psi, y\right)}\right|_{\theta_{-i}=\theta_{-i}^{*}\left(\theta_{i}, \psi\right)}:=\tilde{p}\left(\theta_{i} \mid \psi, y\right),
$$

where $\theta=\left(\theta_{i}, \theta_{-i}\right), \tilde{p}\left(\theta_{-i} \mid \theta_{i}, \psi, y\right)$ is the Gaussian approximation of $p\left(\theta_{-i} \mid \theta_{i}, \psi, y\right)$ and $\theta_{-i}^{*}\left(\theta_{i}, \psi\right)$ is its mode, The approximation in (5) possesses good precision, but it is very time demanding because it requires to recompute $\tilde{p}\left(\theta_{i} \mid \psi, y\right)$ for each value of $\theta$ and $\psi$. A more efficient approach is to use the simplified Laplace Approximation that is based on a Taylor's expansion of $\tilde{p}\left(\theta_{i} \mid \psi, y\right)$ in (5). As mentioned in Rue et al. (2009) and Blangiardo et al. (2013), INLA first explores the marginal joint posterior of the hyperparameters $\tilde{p}(\psi \mid y)$ in order to locate the mode and then performs a grid search to produce a set of "relevant" points $\left\{\psi^{*}\right\}$ together with a set of weights $w_{\psi^{*}}$ as an approximation of this marginal distribution. The marginals $p\left(\psi^{*} \mid y\right)$ are then refined using interpolation methods. Finally, the marginals $\tilde{p}\left(\theta_{i} \mid y\right)$ are obtained as follows:

$$
\tilde{p}\left(\theta_{i} \mid y\right) \approx \sum_{\psi^{*}} \tilde{p}\left(\theta_{i} \mid \psi^{*}, y\right) \tilde{p}\left(\psi^{*} \mid y\right) w_{\psi^{*}}
$$

3.4. Priors. The models proposed in section 3.2 were implemented using the $\mathrm{R}$ package $\mathbf{r}-$ inla (www.r-inla.org). The implementation followed the methods provided in Ruiz-Cárdenas et al. (2012) and Muff et al. (2015) on the use of the INLA methodology in state-space models, dynamic linear models, and in general models whose mean can be written into equation (4).

Like all Bayesian procedures, INLA requires priors for unknown parameters, given below:

- $\alpha_{j}^{i} \sim N(0,3), \beta_{\ell} \sim N(0,3)$ for $i=1, \ldots, N, j=0,1$, and $\ell=0, \ldots, 3$ for Model WF and $\ell=0, \ldots, K(\tau)$ for Model NF. The choice of the variance is completely arbitrary, but the main idea is to select a relatively large one.

- $\rho_{i}:=-\log \sigma_{\epsilon^{i}}^{2} \sim \log$-gamma $\left(1,10^{-20}\right.$ ) (very small precision) for $i=1, \ldots, N$.

- $\rho_{0}:=-\log \sigma_{\eta}^{2} \sim \log$-gamma $\left(1,10^{-20}\right)$ (very small precision). 


\section{Results.}

In the following, the results use model WF for comparison with B14, unless otherwise specified.

4.1. Impact of data reduction choices. As a first exercise, we analyze the change in the predictive capacity of the model when more equations involving proxies are included. We used two proper scoring rules Gneiting and Raftery (2007) as measures of predictive ability: the Interval Score at $\alpha$ level $\left(\mathrm{IS}_{\alpha}\right)$ and the Continuous Ranked Probability Score (CRPS). These scores have been previously employed in the verification of point forecasts in environmental sciences for example, as well as the area of paleoclimatic reconstructions (see B14 and Scheuerer (2014)). Table 2 reports the predictive measures using INLA's prediction intervals and the observed anomalies over 1850-1899 as an out-of-sample validation interval. We used INLA's ability to compute direct posterior densities when computing interval scores and, in the CRPS case, we used Monte Carlo samples obtained with INLA.

Validation to the early instrumental record, important though it is, says little about a reconstruction's behavior on centennial scales, which is of primary interest to climate scientists. To constrain this behavior, we leverage the estimates of Pollack and Smerdon (2004, hereafter, PS04) whose borehole-based temperature inversions estimate surface temperature trends over 1600-1899. Because of the diffusive transfer of heat in Earth's crust, this dataset only speaks to centennial trends; we thus compare it to smoothed versions of our reconstructions, obtained through a Butterworth low-pass filter with a 100-year cutoff and order equal to 4. We compared in this case Model WF with a single RP (the longest available) with respect to model WF using the 8 available reduced proxies, where the comparison was made under the five dimension reduction methods explained above. The results of this comparison are reported as mean square error (MSE) in the rightmost column of Table 2.

An improvement is evident in all measures when we use all the available reduced proxies over the case $N=1$, under the methods SPLS, PCR and sPCR for the first validation period. For the PS04 dataset validation, all the methods except SIR show an improvement in terms of MSE. (Table 2, last column). Also note that, among the models with external 


\begin{tabular}{lll|rrrr|r}
\hline Model & N & Method & IS $_{80}$ & IS $_{95}$ & CRPS & MSE & MSE(PS04) \\
\hline WF & 1 & PCR & 0.4678 & 0.1792 & 0.1641 & 0.0692 & 0.1483 \\
WF & 1 & sPCR & 0.5821 & 0.2146 & 0.2593 & 0.1987 & 0.1981 \\
WF & 1 & LASSO & 0.4658 & 0.1780 & 0.1655 & 0.0714 & 0.1709 \\
WF & 1 & SPLS & 0.4749 & 0.1813 & 0.1810 & 0.0899 & 0.1618 \\
WF & 1 & SIR & 0.4779 & 0.1819 & 0.1671 & 0.0722 & 0.1670 \\
\hline WF & 8 & PCR & 0.2493 & 0.0840 & 0.0986 & 0.0296 & 0.0360 \\
WF & 8 & sPCR & 0.1969 & 0.0719 & 0.0745 & 0.0160 & 0.0892 \\
WF & 8 & LASSO & 0.5044 & 0.3128 & 0.1559 & 0.0462 & 0.1679 \\
WF & 8 & SPLS & 0.3436 & 0.1687 & 0.1177 & 0.0319 & 0.1546 \\
WF & 8 & SIR & 0.6485 & 0.4797 & 0.1871 & 0.0569 & 0.2074 \\
\hline NF & 8 & PCR & 0.2966 & 0.0958 & 0.1249 & 0.0473 & 0.0223 \\
NF & 8 & sPCR & 0.4275 & 0.1624 & 0.1600 & 0.0702 & 0.0237 \\
NF & 8 & LASSO & 0.5434 & 0.3545 & 0.1644 & 0.0491 & 0.1772 \\
NF & 8 & SPLS & 0.2617 & 0.0942 & 0.0967 & 0.0248 & 0.1294 \\
NF & 8 & SIR & 0.5772 & 0.3955 & 0.1720 & 0.0516 & 0.1915 \\
\hline Mixed & 8 & PCR & 0.3509 & 0.1101 & 0.1579 & 0.0745 & 0.0157 \\
Mixed & 8 & sPCR & 0.3660 & 0.1368 & 0.1357 & 0.0512 & 0.0172 \\
Mixed & 8 & LASSO & 0.5291 & 0.3385 & 0.1613 & 0.0480 & 0.1735 \\
Mixed & 8 & SPLS & 0.3131 & 0.1404 & 0.1101 & 0.0294 & 0.1463 \\
Mixed & 8 & SIR & 0.5986 & 0.4209 & 0.1766 & 0.0533 & 0.1969 \\
\hline
\end{tabular}

TABLE 2. Comparison of predictive measures. The IS, CRPS and MSE measures are computed against HadCRUT4 temperature and computed over 18501899 as an out-of-sample validation period. The MSE(PS04) pertains to PS04 and is computed over 1600-1899. All measures are negatively oriented, so lower scores reward better estimates.

forcings, the best performances from the viewpoint of these prediction measures are obtained with sPCR and PCR.

4.2. Impact of Model Choice. We are also interested in assessing whether a linear combination of B-spline bases can model GMST without the inclusion of external forcings at all. It is clear from equations (1) and (3) that one of the drawbacks of Models NF and Mixed is the arbitrariness of $K(\tau)$. We analyze the relationship between the temperature observed during the calibration period (1900-2000) and a linear combination of BSplines. The number of bases in Model NF is selected according to the adjusted $R^{2}$ of a linear regression model between observed anomalies and the corresponding basis functions. Based on the above, 
we selected 6 BSpline functions for this period and we take $K(\tau)=120$ based on the assumption that the number of BSpline bases is uniform throughout the entire reconstruction period. Note that the high prevalence of annually-resolved proxy data allows to assume that a constant number of BSpline bases might be adequate to describe the temperature mean but we know that the location and spacing of knots can possibly affect the results. A compelling alternative to our choice is to use a properly penalized spline with a well defined interpretation in the limit as the number of knots approach infinity. We leave this issue for future investigation..

The choice of $K(\tau)=100$ for model Mixed is based on the same criteria as before. Finally, we fit the models NF and Mixed with the previous choices of $K(\tau)$, using the five different data reduction techniques described in section 3.1. These results are also shown in Table 2. Note that the SPLS method achieves a better performance in terms of the predictive measures for the first validation period, and PCR gets the smallest MSE for the second period.

Four reconstructions of Common Era GMST are shown in Figure 5. In order to illustrate the reconstruction that we obtained for both models WF and Mixed, we considered the best two choices in terms of the validation measures for the first out-of-sample period and the best two choices for the second testing period: sPCR and PCR methods for both the WF and Mixed models.

The best model for the first testing period (sPCR-WF) shows an interesting balance in terms of the variance of the reconstructed series and the width of the confidence region approximated by INLA. The remaining 3 reconstructions show similar small-scale tendencies with respect to the best choice, but the width of their confidence regions is greater. A closer look of the reconstructions is shown in Figure 6. Panel (A), where the first testing period appears between the red lines. Note that sPCR-WF closely predicts the anomalies observed in the first testing period and it does so with a higher level of accuracy than the other reconstructions. In addition, the other three reconstructions underestimate in an almost analogous way the anomalies observed during the same period. At least for this validation 


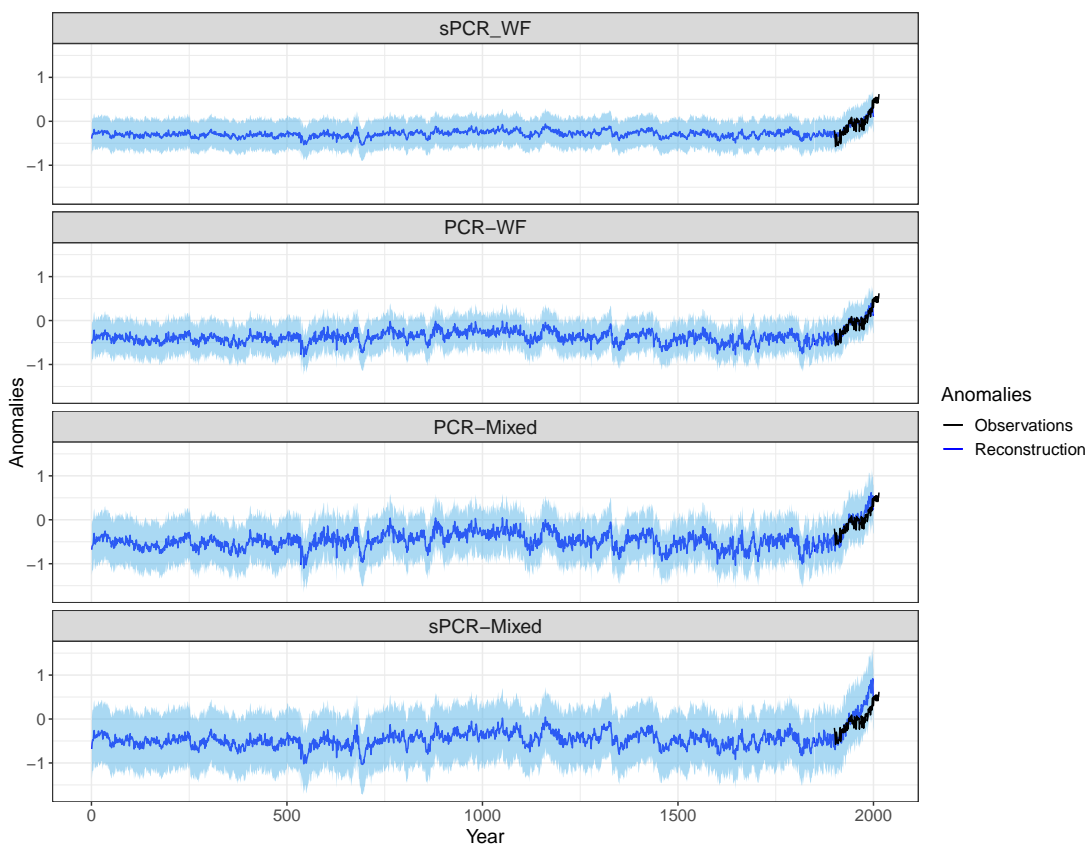

Figure 5. Paleoclimate Reconstruction in the Common Era (CE) with $95 \%$ prediction bands. Best two choices per validation method.

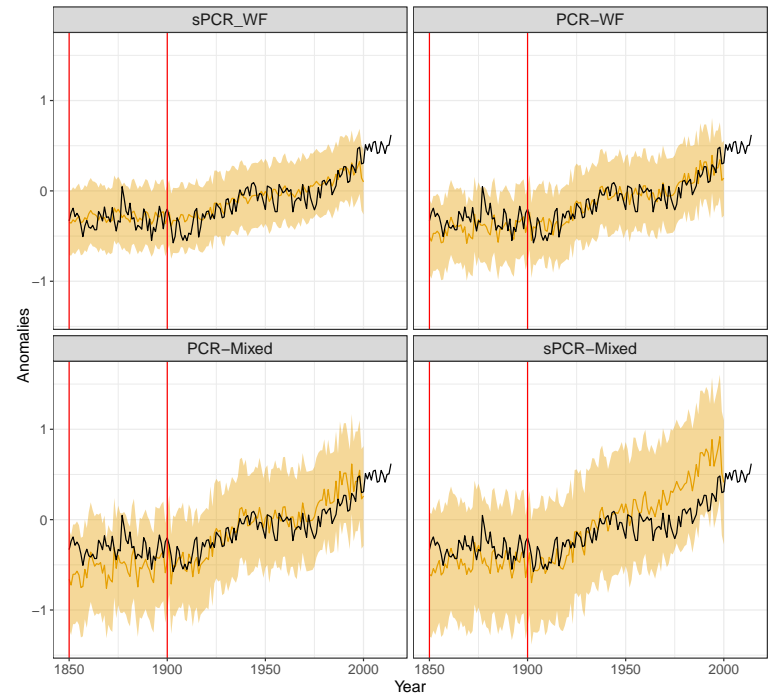

(A)
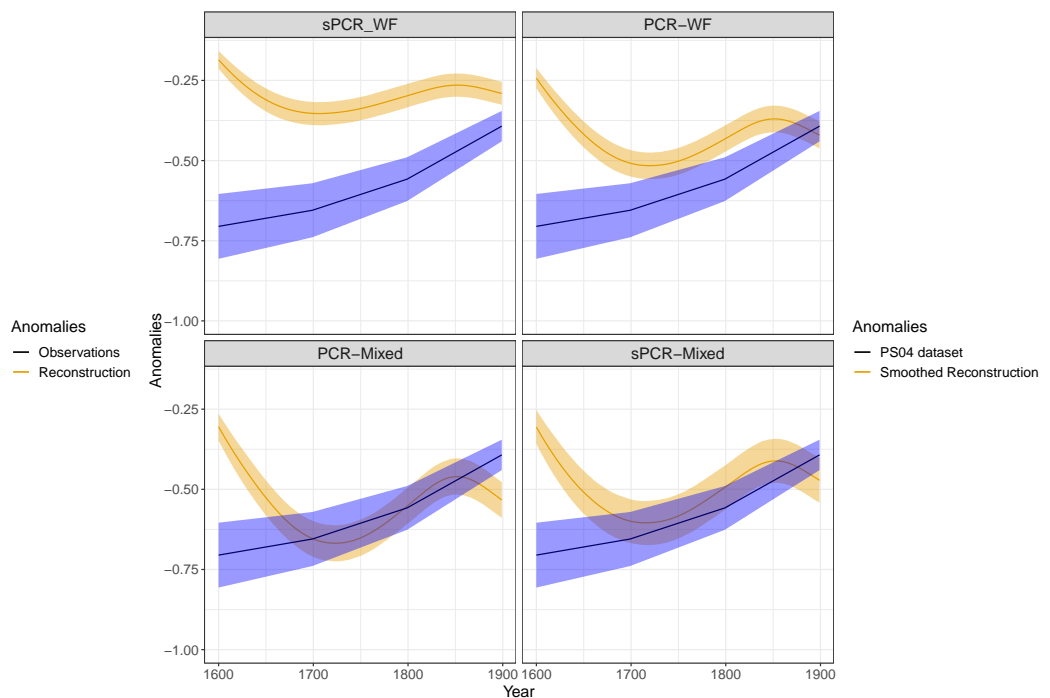

(B)

Figure 6. Panel (A): Paleoclimate Reconstruction 1850-2000 with $95 \%$ prediction bands. The out-of-sample validation period is located between the red lines. Panel (B): Smoothed Reconstruction 1600-1900 vs PS04 series with their corresponding $95 \%$ prediction intervals. Best two choices per validation method. 
exercise there is no clear advantage to using a B-Spline basis as an additional linear term in equation (3).

Figure 6, Panel (B) shows a comparison of the smoothed reconstructions using a low-pass filter and the borehole-based reconstruction of Pollack and Smerdon (2004) (PS04 dataset). In this case, the mixed versions of the sPCR and PCR models with external forcings provide the best adjustment in terms of the MSE measure, especially during the 1700-1850 period. We can infer therefore that the B-Spline basis provides enough flexibility to attain reconstructed anomalies with low-frequency features. Therefore, PCR-Mixed yields the most balanced results in terms of these two validation exercises.

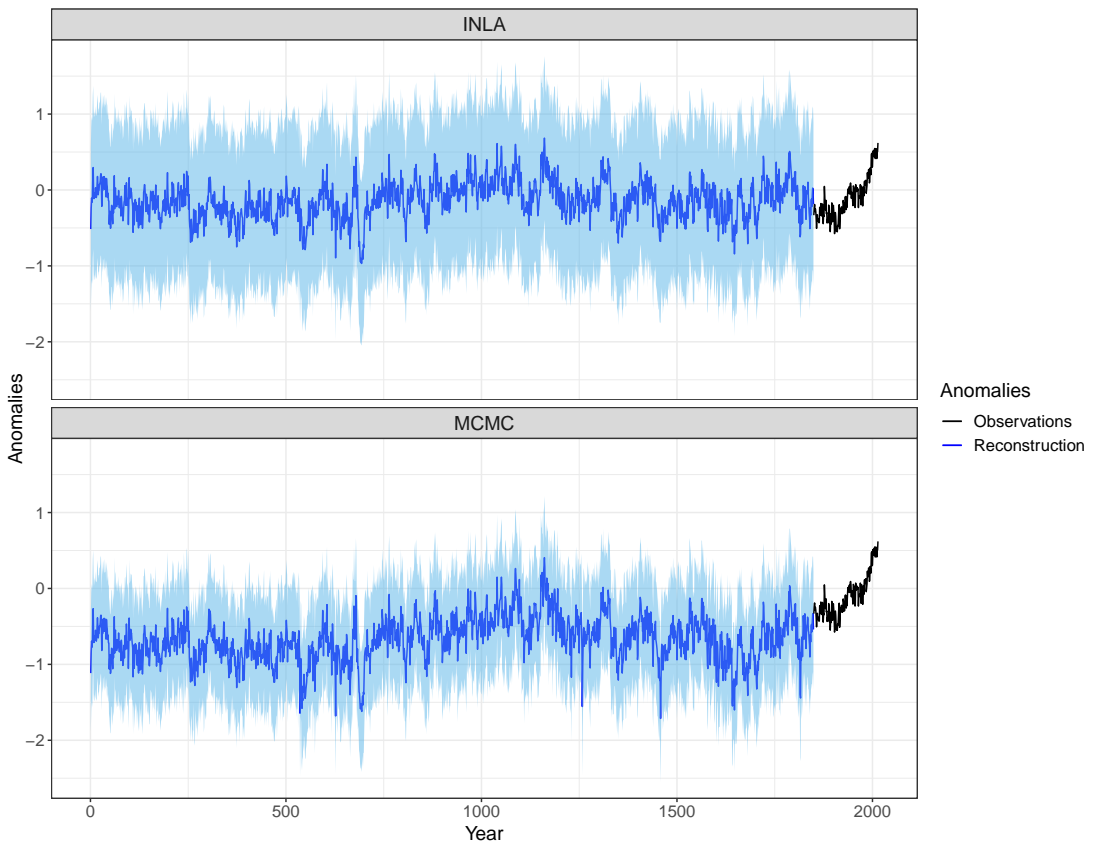

Figure 7. Paleoclimate Reconstruction in the Common Era (CE) with $95 \%$ prediction bands. Model sPCR-WF under two methods: INLA and MCMC.

4.3. Impact of INLA sampling. We now quantify the trade-offs of approximating the MCMC procedure using INLA. The WF model in its simplest case (1 nest) was fitted in B14 using an MCMC approach. We employed this approach in order to adjust the WF model with the first reduced proxy from the sPCR method (chosen purely for comparison purposes). The MCMC was performed using the same priors as in B14, but with a larger calibration period (1900-2000). The results are shown in Figures 7 and 8 . Note that the 

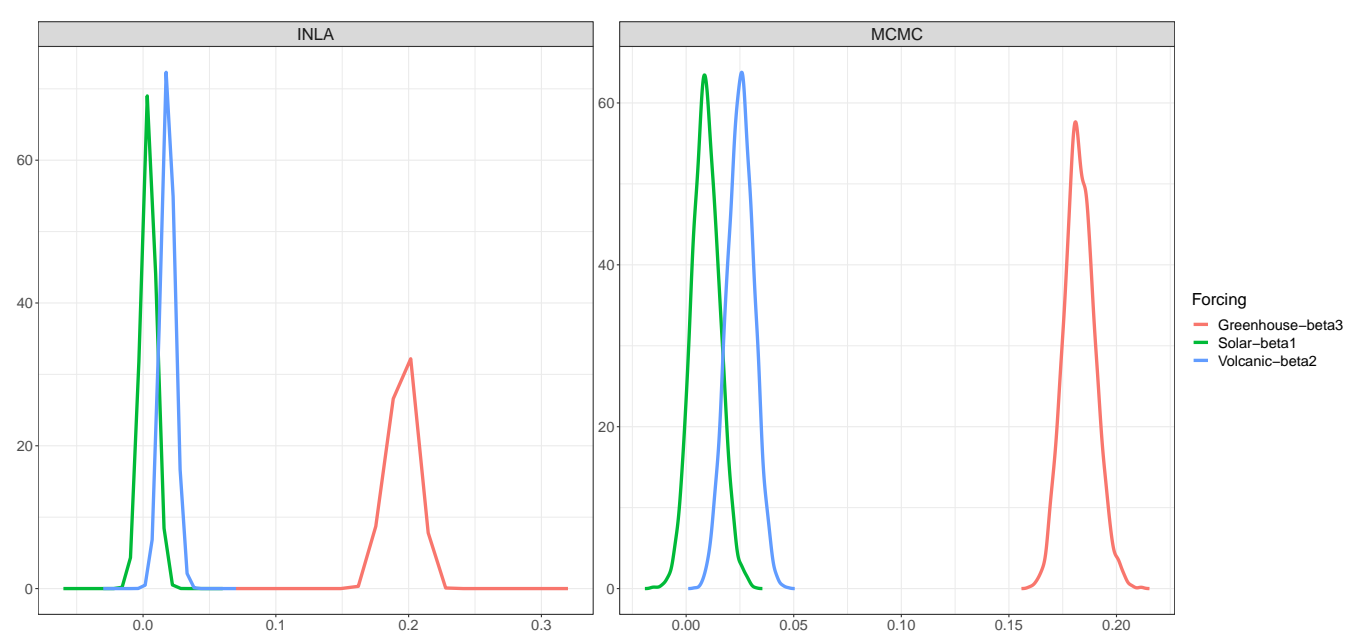

Figure 8. Posterior densities of $\beta_{1}, \beta_{2}$ and $\beta_{3}$ for Model sPCR-WF and MCMC using PCR single reduced proxy.

MCMC reconstruction reaches cooler temperatures than INLA's and that its confidence bands along the reconstruction period are narrower. This last fact coincides with a small difference in terms of the interval score measure. Despite these contrasts, the general trends of both reconstructions are quite similar.

Another point of comparison is the estimated coefficients of the external forcings in equation (2). The estimated density function for each coefficient is shown in Figure 8 . The behavior of the estimated parameters of the three external forcings is very similar between the two methods: by far the most influential external forcing in both reconstructions is the greenhouse gas component, followed by explosive volcanism. Consistent with Schurer et al. (2013b), solar irradiance comes last, and is indistinguishable from a zero effect.

For the same reason as above, the estimated density function of the $\mathrm{CO}_{2}$ coefficients are more concentrated when we use an MCMC algorithm, to a lesser extent for the other coefficients. This behavior confirms the observed details of Figure 7.

In terms of computational efficiency, the INLA procedure is quite remarkable, not only for our case but in most of the previous work on a similar topic as well (see Rue et al. (2009), Blangiardo et al. (2013), Ruiz-Cárdenas et al. (2012) for a few examples). The computational cost of MCMC sampling with 5000 samples was approximately 8 hours, whereas the computational time of INLA's best model with a single reduced proxy was approximately 
15 seconds. This comparison was performed on an Ubuntu 16.04 server with Intel Xeon E5-2630 (8-cores, 2.40GHz) and 64 GB of RAM. This large speedup allowed us to explore a far wider variety of modeling choices than MCMC alone.

\section{Conclusions}

We carried out a Bayesian inference of global mean surface temperature over the past 2,000 years. By leveraging INLA to lighten the computational burden, our framework allows us to investigate a wide range of model choices and data reduction strategies. We validated the result using instrumental data over the 1850-1899 period, and using independent borehole temperature inversions over 1600-1899. The former validates the reconstruction precisely to an interannual scale, while the latter constrains centennial trends.

Below are a few take-home messages:

(a) The data reduction techniques provide roughly equivalent results, with sPCR and PCR performing marginally better than other methods.

(b) The model choice is highly consequential. Model "Mixed" is the most physically justifiable, and it guarantees a balance between the validation measures on the first and second testing periods. This models also appears to perform at least as well as other choices. The additional nonparametric terms in the mean component of equation (3) allows to capture long-memory behavior that is not included in the external forcings, which compensates for the independent structure of the errors.

(c) In cases where both INLA and MCMC are implemented, INLA allows to approximate the MCMC solution at a fraction of the computational cost, but with wider prediction intervals. This exercise was performed only for the simplest model available (1 reduced proxy), because of the prohibitive cost of the MCMC approach for more complex models like the ones presented in Figure 5 .

(d) Adding to a wide body of literature, we find that current temperature levels are unprecedented in the past 2,000 years. Both the models that include forcings (WF, Mixed) show 
that the man-made increase in atmospheric carbon dioxide is the leading contributor to this warming effect.

One limitation of our analysis is the strong dependence of our results on the choice of the testing period. Ideally, a cross-validation exercise should be carried out to determine with greater certainty the expected prediction error of the models, but due to the restricted access to additional comparison information the cross-validation is very challenging for this problem. Another way to improve our current study is to consider the space-time variability of the reduced proxies and temperatures in the model. Since INLA has been proven to be computationally efficient, it could be used to extend this work to a spatiotemporal context. Yet another important area of application is the simultaneous inference of climate fluctuations and deposition age (Sweeney et al., 2018).

\section{REFERENCES}

Bair, E., Hastie, T., Paul, D., and Tibshirani, R. (2006). Prediction by Supervised Principal Components. Journal of the American Statistical Association, 101(473):119-137.

Barboza, L., Li, B., Tingley, M. P., and Viens, F. G. (2014). Reconstructing Past Temperatures from Natural Proxies and Estimated Climate Forcings using Short- and Long-Memory Models. The Annals of Applied Statistics, 8(4):1966-2001.

Benjamini, Y. and Hochberg, Y. (1995). Controlling the false discovery rate: A practical and powerful approach to multiple testing. Journal of the Royal Statistical Society. Series B (Methodological), 57(1):289300.

Blangiardo, M., Cameletti, M., Baio, G., and Rue, H. (2013). Spatial and spatio-temporal models with R-INLA. Spatial and Spatio-temporal Epidemiology, 7:39-55.

Chun, H. and Keleş, S. (2010). Sparse partial least squares regression for simultaneous dimension reduction and variable selection. Journal of the Royal Statistical Society. Series B: Statistical Methodology, 72(1):325.

Chung, D., Chun, H., and Keles, S. (2013). spls: Sparse Partial Least Squares (SPLS) Regression and Classification.

Cook, R. D. (2004). Testing predictor contributions in sufficient dimension reduction. Annals of Statistics, 32(3):1062-1092. 
Coudret, R., Liquet, B., and Saracco, J. (2014). Comparison of sliced inverse regression aproaches for undetermined cases. Journal de la Société Française de Statistique, 155(2):72-96.

Coudret, R., Liquet, B., and Saracco, J. (2017). edrGraphicalTools: Provides Tools for Dimension Reduction Methods.

de Boor, C. (2001). A Practical Guide to Splines. Applied Mathematical Sciences. Springer.

Emile-Geay, J., Cobb, K., Mann, M., and Wittenberg, A. T. (2013). Estimating Central Equatorial Pacific SST variability over the Past Millennium. Part 1: Methodology and Validation. J. Clim., 26:2302-2328.

Fan, J. and Lv, J. (2010). A Selective Overview of Variable Selection in High Dimensional Feature Space. Statistica Sinica, 20:101-148.

Gneiting, T. and Raftery, A. E. (2007). Strictly Proper Scoring Rules, Prediction, and Estimation. Journal of the American Statistical Association, 102(477):359-378.

Guillot, D., Rajaratnam, B., and Emile-Geay, J. (2015). Statistical paleoclimate reconstructions via Markov random fields. Ann. Applied. Statist., pages 324-352.

Hakim, G. J., Emile-Geay, J., Steig, E. J., Noone, D., Anderson, D. M., Tardif, R., Steiger, N., and Perkins, W. A. (2016). The last millennium climate reanalysis project: Framework and first results. Journal of Geophysical Research, 121(12):6745-6764.

Hanhijärvi, S., Tingley, M. P., and Korhola, A. (2013). Pairwise comparisons to reconstruct mean temperature in the Arctic Atlantic Region over the last 2,000 years. Climate Dynamics, 41(7-8):2039-2060.

Hegerl, G. and Zwiers, F. (2011). Use of models in detection and attribution of climate change. Wiley Interdisciplinary Reviews: Climate Change, 2(4):570-591.

Jolliffe, I. T. (1982). A Note on the Use of Principal Components in Regression. Journal of the Royal Statistical Society. Series C (Applied Statistics), 31(3):300-303.

Jones, P. and et al. (2009). High-resolution palaeoclimatology of the last millennium: a review of current status and future prospects. The Holocene, 19(1):3-49.

Jones, P. D., Lister, D. H., Osborn, T. J., Harpham, C., Salmon, M., and Morice, C. P. (2012). Hemispheric and large-scale land-surface air temperature variations: An extensive revision and an update to 2010. Journal of Geophysical Research Atmospheres, 117(5).

Jungclaus, J. H. and et al. (2017). The PMIP4 contribution to CMIP6 - part 3: The last millennium, scientific objective, and experimental design for the PMIP4 past1000 simulations. Geoscientific Model Development, 10(11):4005-4033.

Kennedy, J. J., Rayner, N. A., Smith, R. O., Parker, D. E., and Saunby, M. (2011a). Reassessing biases and other uncertainties in sea surface temperature observations measured in situ since 1850: 1 . Measurement and sampling uncertainties. Journal of Geophysical Research Atmospheres, 116(14):1-13. 
Kennedy, J. J., Rayner, N. A., Smith, R. O., Parker, D. E., and Saunby, M. (2011b). Reassessing biases and other uncertainties in sea surface temperature observations measured in situ since 1850: 2. Biases and homogenization. Journal of Geophysical Research, 116(D14):D14104.

Lee, T. C. K., Zwiers, F. W., and Tsao, M. (2008). Evaluation of proxy-based millennial reconstruction methods. Climate Dynamics, 31:263-281.

Li, B., Nychka, D. W., and Ammann, C. M. (2010). The Value of Multiproxy Reconstruction of Past Climate. Journal of the American Statistical Association, 105(491):883-895.

Li, K.-C. (1991). Sliced Inverse Regression for Dimension Reduction. Journal of the American Statistical Association, 86(414):316-327.

Luterbacher, J., Dietrich, D., Xoplaki, E., Grosjean, M., and Wanner, H. (2004). European seasonal and annual temperature variability, trends, and extremes since 1500. Science, 303(5663):1499-1503.

Mann, M. E., Bradley, R. S., and Hughes, M. K. (1998). Global-scale temperature patterns and climate forcing over the past six centuries. Nature, 392:779-787.

Mann, M. E., Rutherford, S., Wahl, E., and Ammann, C. (2007). Robustness of proxy-based climate field reconstruction methods. Journal of Geophysical Research, 112(D12):D12109+.

Masson-Delmotte, V. and et al. (2013). Information from Paleoclimate Archives. In Stocker, T. F., Qin, D., Plattner, G.-K., Tignor, M., Allen, S., Boschung, J., Nauels, A., Xia, Y., Bex, V., and Midgley, P., editors, Climate Change 2013: The Physical Science Basis. Contribution of Working Group I to the Fifth Assessment Report of the Intergovernmental Panel on Climate Change, pages 383-464, Cambridge, United Kingdom and New York, NY, USA. Cambridge University Press.

McKay, N. P. and Emile-Geay, J. (2016). Technical note: The linked paleo data framework : a common tongue for paleoclimatology. Climate of the Past, 12(4):1093-1100.

Meinshausen, M. and et al. (2017). Historical greenhouse gas concentrations for climate modelling (CMIP6). Geoscientific Model Development, 10(5):2057-2116.

Morice, C. P., Kennedy, J. J., Rayner, N. A., and Jones, P. D. (2012). Quantifying uncertainties in global and regional temperature change using an ensemble of observational estimates: The HadCRUT4 data set. Journal of Geophysical Research Atmospheres, 117(8):1-22.

Muff, S., Riebler, A., Held, L., Rue, H., and Saner, P. (2015). Bayesian analysis of measurement error models using integrated nested Laplace approximations. Journal of the Royal Statistical Society Series C: Applied Statistics, 64(2):231-252.

Olson Hunt, M. J., Weissfeld, L., Boudreau, R. M., Aizenstein, H., Newman, A. B., Simonsick, E. M., Van Domelen, D. R., Thomas, F., Yaffe, K., and Rosano, C. (2014). A variant of sparse partial least squares for variable selection and data exploration. Frontiers in Neuroinformatics, 8(March). 
PAGES2k Consortium (2017). A global multiproxy database for temperature reconstructions of the Common Era. Scientific Data, 4:170088 EP.

Pelletier, J. D. (1998). The power spectral density of atmospheric temperature from time scales of $10^{-2}$ to $10^{6}$ yr. Earth and Planetary Science Letters, 158(3):157-164.

Pollack, H. N. and Smerdon, J. E. (2004). Borehole climate reconstructions: Spatial structure and hemispheric averages. Journal of Geophysical Research D: Atmospheres, 109(11):1-9.

Ramsay, J. and Silverman, B. (2005). Functional Data Analysis. Springer Series in Statistics. Springer, New York, NY, second edition.

Rue, H., Martino, S., and Chopin, N. (2009). Approximate Bayesian Inference for Latent Gaussian Models by Using Integrated Nested Laplace Approximations. Journal of the Royal Statistical Society . Series B ( Methodological ), 71(2):319-392.

Ruiz-Cárdenas, R., Krainski, E. T., and Rue, H. (2012). Direct fitting of dynamic models using integrated nested Laplace approximations - INLA. Computational Statistics and Data Analysis, 56:1808-1828.

Scheuerer, M. (2014). Probabilistic quantitative precipitation forecasting using Ensemble Model Output Statistics. Quarterly Journal of the Royal Meteorological Society, 140(680):1086-1096.

Schneider, T. (2001). Analysis of incomplete climate data: estimation of mean values and covariance matrices and imputation of missing values. Journal of Climate, 14:853-871.

Schurer, A. P., Hegerl, G. C., Mann, M. E., Tett, S. F. B., and Phipps, S. J. (2013a). Separating forced from chaotic climate variability over the past millennium. Journal of Climate, 26(18):6954-6973.

Schurer, A. P., Tett, S. F. B., and Hegerl, G. C. (2013b). Small influence of solar variability on climate over the past millennium. Nature Geoscience, 7:104 EP -.

Smerdon, J. E., Kaplan, A., Chang, D., and Evans, M. N. (2010). A Pseudoproxy Evaluation of the CCA and RegEM Methods for Reconstructing Climate Fields of the Last Millennium. Journal of Climate, 23(18):4856-4880.

Sweeney, J., Salter-Townshend, M., Edwards, T., Buck, C. E., and Parnell, A. C. (2018). Statistical challenges in estimating past climate changes. Wiley Interdisciplinary Reviews: Computational Statistics, 10(5):e1437.

Thomason, L., Vernier, J.-P., Bourassa, A., Arfeuille, F., Bingen, C., Peter, T., and Luo, B. (2015). Stratospheric Aerosol Data Set (SADS Version 2) Prospectus. Technical report.

Tibshirani, R. (1996). Regression Shrinkage and Selection via the Lasso. Journal of the Royal Statistical Society . Series B (Methodological ), 58(1):267-288.

Tierney, L. and Kadane, J. B. (1986). Accurate Approximations for Posterior Moments and Marginal Densities. Journal of the American Statistical Association, 81(393):82-86. 
Tingley, M. P., Craigmile, P. F., Haran, M., Li, B., Mannshardt, E., and Rajaratnam, B. (2012). Piecing together the past: statistical insights into paleoclimatic reconstructions. Quaternary Science Reviews, $35(0): 1-22$.

Tingley, M. P. and Huybers, P. (2013). Recent temperature extremes at high northern latitudes unprecedented in the past 600 years. Nature, 496(7444):201-205.

Toohey, M., Stevens, B., Schmidt, H., and Timmreck, C. (2016). Easy Volcanic Aerosol (EVA v1.0): An idealized forcing generator for climate simulations. Geoscientific Model Development, 9(11):4049-4070.

van Albada, S. and Robinson, P. (2007). Transformation of arbitrary distributions to the normal distribution with application to \{EEG\} test-retest reliability. Journal of Neuroscience Methods, 161(2):205 - 211.

Vieira, L. E. A., Solanki, S. K., Krivova, N. A., and Usoskin, I. (2011). Evolution of the solar irradiance during the Holocene. Astronomy and Astrophysics, 531(A6):1-20.

Wang, J., Emile-Geay, J., Guillot, D., Smerdon, J. E., and Rajaratnam, B. (2014). Evaluating climate field reconstruction techniques using improved emulations of real-world conditions. Climate of the Past, $10(1): 1-19$.

Wu, Q., Liang, F., and Mukherjee, S. (2010). Localized sliced inverse regression. Journal of Computational and Graphical Statistics, 19(4):843-860.

Zou, H. and Hastie, T. (2005). Regularization and variable selection via the elastic net. Journal of the Royal Statistical Society: Series B (Statistical Methodology), 67(5):768-768.

Centro de Investigacion en Matematica Pura y Aplicada (Cimpa)-Escuela de Matematica, Universidad de Costa Rica, San José, Costa Rica

E-mail address: luisalberto.barboza@ucr.ac.cr

Department of Earth Sciences, University of Southern California, Los Angeles, CaliFORNIA, USA.

E-mail address: julieneg@usc.edu

Department of Statistics, University of Illinois at Urbana-Champaign, Champaign, IlliNOIS, USA.

E-mail address: libo@illinois.edu 